\title{
Unique Light-gated Ion Channel Properties of a Novel Modular Cation Channelrhodopsin from an Evolutionary Important Terrestrial Green Alga
}

\section{Rintaro Tashiro}

Kumari Sushmita

Shoko Hososhima

Sunita Sharma

Suneel Kateriya

Hideki Kandori

Nagoya Institute of Technology https://orcid.org/0000-0002-4922-1344

Satoshi Tsunoda ( $\nabla$ tsunoda.satoshi@nitech.ac.jp )

Nagoya Institute of Technology, Showa-ku, Nagoya https://orcid.org/0000-0003-3636-1521

\section{Article}

Keywords: Microbial rhodopsin, channelrhodopsin, electrophysiology, optogenetics, Klebsormidium nitens

Posted Date: September 18th, 2020

DOI: https://doi.org/10.21203/rs.3.rs-68371/v1

License: (1) This work is licensed under a Creative Commons Attribution 4.0 International License.

Read Full License

Version of Record: A version of this preprint was published at Communications Biology on February 23rd, 2021. See the published version at https://doi.org/10.1038/s42003-021-01755-5. 


\section{Abstract}

Channelrhodopsins are a family of microbial rhodopsins that function as a light-gated ion channel. We report the molecular properties of a novel channelrhodopsin KnRh3 from an evolutionary important filamentous terrestrial alga Klebsormidium nitens. KnRh3 is constituted of a 7-transmembrane domain, followed by a long $\mathrm{C}$-terminus moiety that encodes a peptidoglycan binding domain (FimV). When functionally expressed in mammalian cells, KnRh3 showed light-induced cation channel currents. The maximum action spectrum exhibited was at $430 \mathrm{~nm}$ and $460 \mathrm{~nm}$, the former making $\mathrm{KnRh} 3$ one of the most blue-shifted channelrhodopsins characterized thus far. The channel closure rate was relatively fast ( $\tau_{\text {off }}=10 \mathrm{~ms}$ ). Surprisingly, photocurrent kinetics were affected by the C-terminus moiety of KnRh3. When this moiety was truncated to various lengths, this prolonged the channel open lifetime by more than 10fold. We identified two arginine residues, R287 and R291, those are crucial for altering the kinetics. We propose that electrostatic interaction between the 7-TM domain and the $\mathrm{C}$-terminus domain accelerates the photocycle. The most blue-shifted action spectrum of KnRh3 serves as a novel prototype of channelrhodopsin for studying the molecular mechanism of color tuning. In addition, KnRh3 would expand the optogenetics tool kit, especially for when short wavelength excitation is required.

\section{Introduction}

Microbial-type rhodopsins are made up of 7-transmembrane helices with a covalently bound all-trans retinal as a chromophore ${ }^{1}$. These are found in archaea, bacteria, eukaryotes (such as fungi and algae) and viruses and are physiologically responsible for energy production and phototaxis responses. Molecular functions of microbial rhodopsins involve ion transporters, sensors and light-regulated enzymes ${ }^{2,3}$. Ion-transporting rhodopsins are divided into pumps and channels. Bacteriorhodopsin was the first identified outward directed proton-pumping rhodop $\sin ^{4}$. The $\mathrm{Cl}^{-}$pump, the $\mathrm{Na}^{+}$pump, and inwarddirected light-gated proton pumps have also been characterized ${ }^{5-8}$. Detailed structure-function studies together with electrophysiology and molecular dynamics studies revealed the detailed molecular mechanisms of bacteriorhodopsin and other ion-pumping rhodopsins. Channelrhodopsin-1 and - 2 (CrChR1 and CrChR2) from Chlamydomonas reinhardtii were the first light-gated ion channels to be discovered and characterized ${ }^{9,10}$. These proteins permeate cations in which the permeability ratio of $\mathrm{H}^{+}$, $\mathrm{Na}^{+}$and $\mathrm{K}^{+}$is $10^{6}, 1$, and 0.5 , respectively. High-resolution $\mathrm{X}$-ray structures revealed details of the molecular architecture and provided insight into the photoactivation and ion conduction pathway ${ }^{11,12}$. Successful expression of CrChR2 in neurons allowed the action potential to be manipulated by light, which initiated a new research field, optogenetics ${ }^{13,14}$. Channelrhodopsin variants have been engineered to improve the functionality of $\mathrm{ChR}$, and homologous $\mathrm{ChRs}$ were also reported accordingly ${ }^{15}$. Color-tuning variants of ChRs cover almost the entire visible range of the light spectrum. CrChR2 from $C$. reinhardtii exhibits an action spectrum maximum at $470 \mathrm{~nm}^{10}$. ChR variants such as $\mathrm{C} 1 \mathrm{~V} 1$, which is the chimeric version of ChR1 from $C$. reinhardtii and Volvox carteri, or C1C2 (green light receiver) absorb around $530 \sim$ $545 \mathrm{~nm}$ light ${ }^{16,17}$. A further red-shifted ChR, Chrimson from Chlamydomonas noctigama, exhibits an 
absorption maximum at $590 \mathrm{~nm}$ which allows reliable neuronal stimulation over $600 \mathrm{~nm}$ light when applied to neurons ${ }^{18}$. On the other hand, TsChR or PsChR absorb shorter wavelengths, making it possible to excite neurons at $440 \mathrm{~nm}^{19}$. The open channel lifetime can be extended by mutations at $\mathrm{C} 128$ and D156 (DC pair) which form a hydrogen bond bridge in CrChR2. A mutation at C128 to T, A and S slowed channel closure kinetics by $1000-$ fold $^{20}$. The CrChR2 D156C has an even stronger effect, with high light sensitivity and a prolonged open channel lifetime, up to $30 \mathrm{~min}^{21}$. Recent genomic database research reveals that various lower eukaryotes contain opsin-related genes. Consequently, a number of ChR genes were identified and their molecular properties, such as ion selectivity and absorption maxima, were shown to be different.

Recently, the genome of an evolutionary important terrestrial green alga (Klebsormidium flaccidum) was sequenced, and analysis of the genome delineated the molecular basis of the adaptation of land plants ${ }^{28}$. K. flaccidum was renamed as Klebsormidium nitens (http://www.plantmorphogenesis.bio.titech.ac.jp/ algae_genome_project/klebsormidium/). K. nitens adapts to diverse environmental conditions such as desiccation, light, and temperature gradients ${ }^{29}$. However, the sensory photoreceptor landscape of $K$. nitens has not yet been established. For the first time, we identified a ChR homologue that encodes a novel modular channelrhodopsin from an evolutionary important filamentous terrestrial alga, $K$. nitens. A unique feature of $\mathrm{KnRh} 3$ is that it includes the FimV domain at the C-terminus of rhodopsin. FimV is involved in peptidoglycan-binding protein which is responsible for twitching motility in Pseudomonas aeruginosa and other microbes ${ }^{30}$. Thus, it is anticipated that light signals are transduced via ChR into motility in $K$. nitens.

In this report, we performed an electrophysiological experiment using KnRh3 to reveal its ion channel function. Its photocurrent properties such as channel kinetics, absorption maximum, light sensitivity and ion selectivity was compared to those of CrChR2. For the first time, we show an unexpected modulation of the photocurrents of KnRh3 by its extended C-terminus region. Novel KnRh3 has the potential to expand the optogenetics tool kit, especially for dual light (two photon) applications when short wavelength excitation is required. Moreover, functional characterization of channelrhodopsin from an evolutionary terrestrial alga indicates the existence of channelrhodopsin-mediated physiological responses beyond aquatic habitats (marine and freshwater systems).

\section{Materials And Methods}

\section{Expression plasmids}

Synthesized genes encoding KnRh3 and CrChR2 (1-315 amino acids) were subcloned into a phKR2-3.0eYFP plasmid (gifted by Dr. H. Yawo (University of Tokyo, Japan)) using an In-Fusion HD cloning kit (Takara Bio, Shiga, Japan). Length variants of KnRh3 (amino acid length of 272, 280, 290, 300, 310, 317 , $321,397,697$, and 831 ) were created by using appropriate PCR primers. For immunostaining experient, the plasmid pKnRh3(697 amino acids)-3.0-eYFP was inserted N-QKLISEEDL-C (10 amino acids, c-Myc 
epitope tag) in the C-terminal of eYFP using inverse PCR. Site-directed mutagenesis was performed using a QuikChange site-directed mutagenesis kit (Agilent, CA, USA). All the constructs were verified by DNA sequencing (Fasmac Co., Ltd. Kanagawa, Japan).

\section{Mammalian cell culture}

Electrophysiological assays and immunostaining of $\mathrm{KnRh} 3$ and $\mathrm{CrChR} 2$ were performed on ND7/23 cells, which are hybrid cell lines derived from neonatal rat dorsal root ganglia neurons fused with mouse neuroblastoma. ND7/23 cells were grown on a coverslip in Dulbecco's modified Eagle's medium (DMEM; Wako, Osaka, Japan) supplemented with $2.0 \mathrm{mM}$ of all-trans retinal and $5 \%$ fetal bovine serum, and under a $5 \% \mathrm{CO}_{2}$ atmosphere at $37^{\circ} \mathrm{C}$. The expression plasmids were transiently transfected by using the FuGENE HD transfection Reagent (Promega, Fitchburg, WI, USA) according to the manufacturer's instructions. Electrophysiological recordings were then conducted 24-36 $\mathrm{h}$ after transfection. Successfully transfected cells were identified by eYFP fluorescence under a microscope prior to measurements.

\section{Immunostaining of mammalian cells}

ND7/23 cells were cultured on glass coverslips. Cells expressing KnRh3 or KnRh3 bearing the c-Myc epitope tag at the C-terminus were fixed in $4 \%$ paraformaldehyde phosphate buffer solution for $15 \mathrm{~min}$ at room temperature. Cells were then washed with phosphate-buffered saline (PBS) for three times. When necessary, cells were permeabilized with $0.5 \%$ Triton X-100 for $15 \mathrm{~min}$ at room temperature. Cells were treated with blocking buffer consisting of $3 \%$ goat serum for $60 \mathrm{~min}$ at room temperature. After blocking, the cells were incubated with rabbit anti-c-Myc primary antibody (C3956; Sigma-Aldrich, St. Louis, MO, USA) at a 1:500 dilution for 60 min at room temperature, then washed with PBS for three times and labeled with goat anti-rabbit IgG secondary antibody Alexa Fluor 594 (A-11037; Thermo Fisher Scientific, Waltham, MA, USA) at a 1:200 dilution for $2 \mathrm{~h}$ at room temperature. After a final wash with PBS, the coverslips were mounted on glass slides with ProLong Diamond Antifade Mountant (Thermo Fisher Scientific).

Live cultured KnRh3 bearing the c-Myc epitope tag at the C-terminus expressing-cells were washed with PBS. The cells were moved from the culture medium to serum-free DMEM and incubated with rabbit anti-C-Myc primary antibody at a 1:500 dilution for $60 \mathrm{~min}$ under a $5 \% \mathrm{CO}_{2}$ atmosphere at $37^{\circ} \mathrm{C}$. The cells were washed with PBS twice before fixing with 4\% paraformaldehyde phosphate buffer solution for 15 min at room temperature. The cells were washed with PBS three times before labeling with goat antirabbit IgG secondary antibody Alexa Fluor 594 at a 1:200 dilution for $2 \mathrm{~h}$ at room temperature. After a final wash with PBS, the coverslips were mounted on glass slides with ProLong Diamond Antifade Mountant.

Fluorescent images were acquired with a confocal microscopy (LSM880, CarlZeiss, Jena, Germany) equipped with $\times 40$ objective lens. 


\section{Electrophysiology.}

All experiments were carried out at room temperature $\left(22 \pm 2^{\circ} \mathrm{C}\right)$. Photocurrents were recorded by an Axopatch 200B amplifier (Molecular Devices, Sunnyvale, CA, USA) under a whole-cell patch clamp configuration ${ }^{14}$. Data were filtered at $5 \mathrm{kHz}$ and sampled at $20 \mathrm{kHz}$ (Digdata1550, Molecular Devices, Sunnyvale, CA, USA) and stored in a computer (pClamp10.7, Molecular Devices). Pipette resistance was 3-6 M . The standard internal pipette solution for the whole-cell voltage clamp contained (in mM) 126 NaAsp, $0.5 \mathrm{CaCl}_{2}, 2 \mathrm{MgCl}_{2}, 5 \mathrm{EGTA}, 25 \mathrm{HEPES}, 12.2 \mathrm{NMG}$, and adjusted to $\mathrm{pH} 7.4$ by citric acid. The standard extracellular solution for the whole-cell voltage clamp contained (in $\mathrm{mM}$ ) $150 \mathrm{NaCl}, 1.8 \mathrm{CaCl}_{2}, 1$ $\mathrm{MgCl}_{2}$, and $10 \mathrm{HEPES}, 10 \mathrm{NMG}, 5$ glucose, and adjusted to $\mathrm{pH} 7.4$ by NMG. Internal and external solutions for ion selectivity are shown in Table 1 . The liquid junction potential was calculated and compensated by pClamp 10.7 software. Time constants were determined by a single exponential fit unless indicated otherwise.

Table 1

Measurment solution component for ion selectivity

\begin{tabular}{ll|cccccccc} 
& & $\mathrm{NaCl}$ & $\mathrm{KCl}$ & $\mathrm{CaCl}_{2}$ & $\mathrm{MgCl}_{2}$ & $\mathrm{NMG}$ & $\mathrm{EGTA}$ & $\mathrm{CHES}$ & $\mathrm{MES}$ \\
\hline Pipette & $\mathrm{NMG} \mathrm{pH9.0}$ & 1 & 1 & 2 & 2 & 140 & 10 & 10 & - \\
\hline Bath & $\mathrm{NMG} \mathrm{pH9.0}$ & 1 & 1 & 2 & 2 & 140 & - & 10 & - \\
& $\mathrm{NMG} \mathrm{pH6.85}$ & 1 & 1 & 2 & 2 & - & - & - & 10 \\
& $\mathrm{Na}{ }^{+} \mathrm{pH} 9.0$ & 140 & 1 & 2 & 2 & - & - & 10 & - \\
& $\mathrm{K}^{+} \mathrm{pH} 9.0$ & 1 & 140 & 2 & 2 & - & - & 10 & - \\
& $\mathrm{Ca}^{2+} \mathrm{pH} 9.0$ & 1 & 1 & 70 & 2 & - & - & 10 & - \\
& $\mathrm{Mg}^{2+} \mathrm{pH} 9.0$ & 1 & 1 & 2 & 70 & - & - & 10 & -
\end{tabular}

\section{Optics}

For whole-cell voltage clamp, irradiation at $480 \mathrm{~nm}$ was carried out using collimated LED (parts No. LCS0470-03-22, Mightex, Toronto, Canada) controlled by computer software (pCLAMP10.7, Molecular Devices). Light power was measured directly by an objective lens of a microscope by a power meter (LP1, Sanwa Electric Instruments Co., Ltd., Tokyo, Japan). All action spectra were measured at the same light intensity in the range of $410 \mathrm{~nm}$ to $650 \mathrm{~nm}$ by a xenon light source OSG (Hamamatsu photonics, Hamamatsu, Japan). 


\section{Statistical validation of the data}

Data in the text and figures are expressed as mean \pm SEM and were evaluated with the Mann-Whitney $U$ test for statistical significance, unless noted otherwise. Means were judged as statistically insignificant when $\mathrm{P}>0.05$.

\section{Results}

\section{Genome of terrestrial alga encodes a novel modular channelrhodopsin (KnRh3)}

Extensive and targeted mining of the genomic database of $K$. nitens revealed the existence of several rhodopsin-encoding genes (http://www.plantmorphogenesis.bio.titech.ac.jp /cgibin/ blast/blast_www_klebsormidium.cgi). Among them, we found one gene that is highly homologous to chlorophyte cation channelrhodopsins such as $\mathrm{CrChR2}$. We named it KnRh3 in this study. KnRh3 possesses 831 amino acids and harbors a membrane-embedded 7-TM rhodopsin domain (amino acids 1-272) followed by a long C-terminus region (273-831 amino acids) (Fig. S1). Sequence comparison of the rhodopsin domain revealed $34.5 \%$ identity with and $69.3 \%$ homology to CrChR2. Several important amino acid residues were aligned when compared to four ChRs and bacteriorhodopsin (BR) (Fig. 1A). Three (Glu82, Glu90, Glu97) out of five glutamate residues at positions 82, 83, 90, 97 and 101 in CrChR2 that are important for channel activity were conserved in KnRh3. Glu83 was replaced by Val. Glu101 was substituted by Pro in KnRh3 whereas in GtACR1 (light-gated anion channel), Gln was observed at this position. His instead of Asp at position 96 was regarded as a characteristic of channelrhodopsins, but in $\mathrm{KnRh} 3$, this position was occupied by Ala, by D156 in GtACR1, while C128 in CrChR2 formed a hydrogen bridge (D-C pair or D-C gate) which altered the channel open lifetime ${ }^{20,21}$. This pair is also conserved in KnRh3 whereas chlorophyte cation channel rhodopsin (GtCCR4) lacks it. Thus, we predicted that KnRh3 might function as a cation channelrhodopsin when functionally expressed in a suitable system. Notably, $\mathrm{KnRh} 3$ has a long C-term domain that encodes a putative peptidoglycan binding domain (FimV) between 410-690 amino acids (Fig. S1). In general, the peptidoglycan layer is located outside of the cytoplasmic membrane in bacterial cell walls. In fact, the homologous region of $\mathrm{KnRh} 3$ is predicted to be located on the extracellular side, whereas the $\mathrm{C}$-terminus region is normally located on the cytoplasmic side in the case of microbial-type rhodopsin (Fig. S2). Thus, we considered why the FimV domain is connected to the C-terminus of the 7-TM domain. We anticipated that the membrane topology of $\mathrm{KnRh3}$ is inverted, as in the case of heliorhodopsin (HeR), which is a recently identified subfamily in microbial rhodopsins ${ }^{31}$. We synthesized a codon-adapted full-length gene of KnRh3 (831 amino acids) for functional expression in a mammalian system and fused it with the p3.0-eYFP vector with eYFP at the C-terminus of KnRh3. After transfection of the fusion expression construct, we observed weak YFP fluorescence on the cellular membrane, indicating poor expression in ND7/23 cells. A voltage-clamp measurement was then performed. However, no photocurrent was observed (data not shown). We anticipated that low expression was due to a long C-terminus domain ( 540 amino acids) after the 7-TM domain. Thus, we 
systematically truncated the C-terminus to various lengths. We created five variants with different lengths, carrying $697,397,310,290$, and 272 amino acids. Note that only the variant with 697 amino acids included the FimV domain. In addition, we fused a cMyc epitope tag at the C-terminus of the 697 amino acids variant to detect immune fluorescence. Figure 1B-D shows fluorescent images of the transfected cells. eYFP fluorescence indicates successful expression of KnRh3. Although no alexa595 signal was observed in the absence of detergent (Fig. 1B), treatment with detergent to permeabilize the membrane generated a signal (Fig. 1C). No alexa595 signal was observed when KnRh3 without cMyc-tag was expressed even after the detergent treatment (Fig. 1D). These results indicate that the $\mathrm{C}$-terminus domain is on the cytoplasmic side. Thus, KnRh3 exhibits typical membrane topology of a channelrhodopsin.

\section{The C-terminus of KnRh3 modulates photocurrents and photocycle of the channelrhodopsin domain}

We then performed patch clamp recordings of each variant. All the constructs having various amino acid lengths are depicted in Fig. 2A. As shown in Fig. 2B-F, large photocurrents were observed in all the five $\mathrm{KnRh} 3$ variants upon blue light illumination for 1.0 second. Photo-current amplitudes were comparable to that of CrChR2 (Fig. 2G). Current direction reversed as clamped voltage shifted from - 90 to $+50 \mathrm{mV}$ in $20 \mathrm{mV}$ steps, which is a typical ion channel property. Illumination induced a large peak current (Ip) which slowly decayed into smaller amplitudes during illumination. However, the current did not reach a plateau within $1 \mathrm{sec}$, whereas the photocurrent of CrChR2 rapidly dropped to a steady state level (Fig. 2G). When light was shut off, this diminished the photocurrent into the baseline, indicating channel closure. Notably, the phases of current decay were significantly different among the five variants, two of which, those with 697 and 397 amino acids, rapidly decayed into the baseline whereas the other three (300, 290 and 272 amino acids) showed a relatively slow decay (Fig. 2B-F). This indicates that the channel kinetics and the photocycle are affected by the cytoplasmic domain of KnRh3. To further investigate the observation above, we created in addition four variants with different lengths of amino acids: 321, 317, 310 and 280. Current decay kinetics ( $\tau$-off) at $-70 \mathrm{mV}$ from all nine variants were compared (Fig. $2 \mathrm{H}$ ). The variants longer than 317 amino acids showed a fast off-kinetics ( $10 \mathrm{~ms})$. The variant with 310 amino acids showed an off-kinetics of about $20 \mathrm{~ms}$. Furthermore, the time constants of the shorter variants became even larger and the shortest variant with 272 amino acids reached $130 \mathrm{~ms}$, more than 10 times slower than the original construct. This result clearly shows a correlation between the length of amino acids and the time constant of channel kinetics in $\mathrm{KnRh} 3$, indicating that the $\mathrm{C}$-terminal cytoplasmic domain affects both channel kinetics and the photocycle. In particular, amino acids between 272 and 310 largely contribute to the modulation of light-gated ion channel activities in $\mathrm{KnRh3}$.

Interestingly, the photocurrent amplitudes also differed among the nine variants (Fig. 2I). The variants shorter than 300 amino acids reached about $3 \mathrm{nA}$ at $-70 \mathrm{mV}$, while the longer variants $>310$ amino acids showed smaller currents under the same condition in which only about 1 nA was observed from cells expressing the 397 and 697 amino acids variants. We reasoned that current amplitude depends on the open-life time of the channels. As shown in Fig. $2 \mathrm{H}$, the shorter variants exhibited a larger time constant. 
This implies that these variants have a stable M-intermediate that corresponds to the channel's open state, according to a previous study on $\mathrm{CrChR2}{ }^{32}$. Thus, the apparent current amplitude of the short variants was elevated as the M-state accumulated during illumination.

The current-voltage relations (I-V plots) of KnRh3 and CrChR2 are depicted in Fig. $2 \mathrm{~J}$ (peak component) and Fig. 2K (steady state component). Current direction and amplitude depend on the applied voltage. The reversal potential of $\mathrm{KnRh} 3$ is about $-10 \mathrm{mV}$ while that of $\mathrm{CrChR2}$ is $0 \mathrm{mV}$ under the same condition. As is already known, ion conductance of CrChR2 is inwardly-rectified and thus the outward current was largely suppressed (Fig. $2 \mathrm{~J}$ and $\mathrm{K}$, black). The $\mathrm{I}-\mathrm{V}$ plots of $\mathrm{KnRh} 3$ show a rather weak rectification and relatively large outward currents were observed (see $+50 \mathrm{mV}$ in Fig. $2 \mathrm{~J}$ and $\mathrm{K}$ ). The I-V plots of all the variants are summarized in Fig. S3. The photocurrent properties of KnRh3 397 amino acids (excluding FimV) and 697 amino acids (including FimV) are shown in Fig. S4. The current shape, I-V relation, and current amplitudes of these two variants were identical. These results indicate that FimV did not alter channel function.

All nine variants that were tested were fused to eYFP at the C-terminal. Thus, one might argue whether the length-dependent effect in Fig. 21 might have originated from eYFP, i.e. if an interaction between rhodopsin and eYFP may have altered the channel kinetics for an unknown reason. To exclude this possibility, we created 272 amino acids and 397 amino acids KnRh3 variants without eYFP. The variants were transfected, and the photocurrents were recorded to compare the channel-off kinetics and amplitude (Fig. 3). The time constants (t-off) were almost identical between eYFP-tagged and untagged 272 amino acids and 397 amino acids variants (Fig. 3A). This clearly indicates that a length-dependent effect was observed, regardless of the presence of eYFP at the C-terminus of KnRh3. Photocurrent amplitudes of untagged variants were smaller than those of eYFP-tagged constructs (Fig. 3B). This could be due to a lower expression level of the former. In fact, eYFP constructs contain the ER-exporting signal peptide and the membrane trafficking signal peptide to improve membrane localization, whereas no additional sequence was tagged in the untagged variants.

\section{Positively charged residues in the C-terminus domain of $\mathrm{KnRh} 3$ regulated intramolecular interactions}

Based on the result in which the cytoplasmic domain altered the channel kinetics in KnRh3 (Fig. 2H), it could be hypothesized that the cytoplasmic domain interacts with the rhodopsin domain (7-TM domain). Since no significant change in t-off was observed among variants longer that 317 amino acids, the region from 317 amino acids onwards is not important for the interaction. Thus, we anticipated that one or several amino acids in the cytoplasmic region between 272 and 317 amino acids would be crucial for the interaction. We noticed two characteristic negatively charged residues, E285 and E293, and three positively charged residues, R287, K289 and R291, some of which might form an electrostatic interaction with the 7-TM domain (Fig. 4A and Fig. S1). Thus, we replaced each residue with alanine in the KnRh3397 amino acids variant which exhibits fast kinetics, similar to the longest variant (697 amino acids). As shown in Fig. 4B-D, photocurrent decay after switching off light apparently became slower after mutating 
R287A and R291A. The decay was further slowed in the double mutant R287A/R291A (Fig. 4E). Figure 4F summarizes the time constants of all the mutants tested. The R287A mutant shows a t-off of $50 \mathrm{~ms}$ which is five-fold slower than that of the KnRh3-397 variant, while R291A was slowed down to $25 \mathrm{~ms}$. The double mutant R287A/R291A reached about 100 ms which is more than 10 times slower than KnRh3-397 amino acids (no amino acid substitution) and is close to the 272 amino acids (no amino acid substitution) variant (130 ms). The three remaining mutants (E285A, K289A and E293A) showed no effect on kinetics. These results clearly indicate that the positively charged residue, R287 and R291, interact with the 7-TM domain and contribute to altered channel kinetics and the photocycle (Fig. 4I). These three mutants exhibited significantly larger photocurrents than KnRh3-397 (Fig. 4G) without any change in their reversal potential (Fig. 4H).

\section{Action spectrum and light sensitivity of $\mathrm{KnRh3}$}

The action spectrum, a wavelength dependency of photocurrent amplitude, was measured with a multicolor light source (Fig. 5). The 272 amino acids and 397 amino acids variants showed a spectrum with two $\lambda_{\max }$ at 430 and $460 \mathrm{~nm}$ which are shorter than that of $\operatorname{CrChR2}\left(\lambda_{\max }=470 \mathrm{~nm}\right)$. To our knowledge, the $\lambda_{\max }$ of KnRh3 is one of the shortest among naturally occurring ChRs.

We then measured the light-power dependency of two KnRh3 variants (272 amino acids and 397 amino acids), comparing them to CrChR2 (Fig. 6). Photocurrent amplitude, including Ip (peak component) and Is (steady state component), grew as light intensity increased. The Is of the 272 variant became saturated at about $0.09 \mathrm{~mW} / \mathrm{mm}^{2}$ (Fig. 6A empty circle), while Ip increased further and reached a plateau at about $0.2 \sim 03 \mathrm{~mW} / \mathrm{mm}^{2}$ (Fig. 6A filled circle). On the other hand, light sensitivity of the 397 amino acids variant was markedly lower than that of the 272 amino acids varint. Both Ip and Is became saturated at about 3$4 \mathrm{~mW} / \mathrm{mm}^{2}$ (Fig. 6B). Note that the x-axis of each panel differs by a single order of magnitude. The lightpower dependency of CrChR2 falls in the same range as KnRh3 397 amino acids (Fig. 6C). The halfsaturation light intensity $\left(\mathrm{EC}_{50}\right)$ was determined to be $0.051 \mathrm{~mW} / \mathrm{mm}^{2}(\mathrm{KnRh} 3-272), 0.712 \mathrm{~mW} / \mathrm{mm}^{2}$ (KnRh3-397) and $0.731 \mathrm{~mW} / \mathrm{mm}^{2}$ (CrChR2). This indicates that the C-terminal length affected the light sensitivity of $\mathrm{KnRh3}$.

\section{$\mathrm{KnRh} 3$ permeates monovalent and divalent cations}

We predicted that $\mathrm{KnRh} 3$ functions as a cation channelrhodopsin considering its sequence similarity to chlorophyte channelrhodopsins (e.g. CrChR2). Ion selectivity was tested by patch-clamp recording. We used the KnRh3 272 amino acids variant for all experiments. The extracellular solution was systematically replaced with various cations, while the intracellular solution was fixed (refer to Table 1 for more details). Figure 7A-F shows representative photocurrent traces under various ionic conditions. In the presence of NMG, we assumed that $\mathrm{H}^{+}$was the conducting ion. The I-V plot under several ionic conditions is depicted in Fig. 7G (KnRh3) and Fig. 7H (CrChR2). Note that the peak component (Ip) of the photocurrent was plotted for all the relevant measurements, and essentially the same result was obtained 
from the analysis of Is, which is summarized in Fig. S5 and S6. When Fig. 7A and 7B are compared, voltage dependency of current direction was obviously different. The reversal potential was shifted from $-30 \mathrm{mV}$ to $+20 \mathrm{mV}$ (Fig. $7 \mathrm{G}$ red and blue), indicating $\mathrm{H}^{+}$conductance across KnRh3 (Fig. 7G, I, J and K). Large $\mathrm{Na}^{+}$conductance was observed, as shown in Fig. 7C, with a reversal potential of $+20 \mathrm{mV}$ (Fig. $7 \mathrm{G}$ green, $\mathrm{J}$ and $\mathrm{K}$ ). The shift in reversal potential of the $\mathrm{K}^{+}$solution indicates that $\mathrm{KnRh} 3$ permeates $\mathrm{K}^{+}$ (Fig. 7l, J and K Fig. S5). We then tested divalent cations, $\mathrm{Ca}^{2+}$ and $\mathrm{Mg}^{2+}$. We observed an inward photocurrent in the $\mathrm{Ca}^{2+}$ solution (Fig. 7E) and reversal potential shifted significantly from - $30 \mathrm{mV}$ (NMG $\mathrm{pH}$ 9.0) to $-5 \mathrm{mV}\left(\mathrm{Ca}^{2+} \mathrm{pH}\right.$ 9.0) (Fig. $7 \mathrm{G}$ red and black, J, K). A similar observation was observed in the $\mathrm{Mg}^{2+}$ solution (Fig. 7F, I, J and 7K, Fig. S5). Altogether, we demonstrated that KnRh3 is a cation channelrhodopsin that permeates monovalent and divalent cations. Figure 7H shows the I-V plot of Cr_ChR2 under the same condition as Fig. 7G. Reversal potentials and a shift in reversal potential ( $\Delta$ Erev) of $\mathrm{KnRh} 3$ and $\mathrm{CrChR2}$ are depicted in Fig. $7 \mathrm{~J}, 7 \mathrm{~K}$ and $7 \mathrm{~L}$, respectively. These values allow the permeability ratio of each cation to be estimated. The $\Delta$ Erev of $\mathrm{H}^{+}, \mathrm{Na}^{+}, \mathrm{K}^{+}, \mathrm{Ca}^{2+}$ and $\mathrm{Mg}^{2+}$ from $\mathrm{CrChR} 2$ were determined as $90,70,65,40$ and $35 \mathrm{mV}$, respectively (Fig. 7L). This indicates that the rank in permeability ratio is $\mathrm{H}^{+}>\mathrm{Na}^{+}>\mathrm{K}^{+}>\mathrm{Ca}^{2+}>\mathrm{Mg}^{2+}$. On the other hand, $\mathrm{KnRh} 3$ showed no significant difference in $\Delta \mathrm{Erev}$ among $\mathrm{H}^{+}, \mathrm{Na}^{+}$and $\mathrm{K}^{+}(45 \sim 48 \mathrm{mV})$, indicating that permeability of these cations was almost the same (Fig. 7K). The $\Delta$ Erev of $\mathrm{Ca}^{2+}$ and $\mathrm{Mg}^{2+}$ were about 30 and $25 \mathrm{mV}$, respectively, indicating lower permeability for divalent cations than monovalent cations (Fig. 7K). As mentioned above, we analyzed the peak component of each photocurrent. In addition, Is was analyzed. Essentially the same results were found (Fig. S6).

\section{Discussion}

In this study, we report on the electrophysiological characterization of a newly identified multi-domain cation channelrhodopsin from Klebsormidium nitens (KnRh3). KnRh3 possesses a large cytoplasmic domain made of about 540 amino acids which encode a peptidoglycan binding moiety (FimV). Several ChRs also have a long C-terminus tail. Full-length ChR1 and ChR2 from Chlamydomonas reinhardtii contain 712 and 737 amino acids in total (837 and 747 in Volvox carteri). Among them, the 7-TM rhodopsin domain is made up of only about 300 amino acids. Thus, $400-500$ amino acids are located mainly on the cytoplasmic side. However, no significant homology was found among the long cytoplasmic extension of the characterized ChRs from these different organisms. Since the discovery of $\mathrm{ChRs}$, the role of cytoplasmic domains remains barely understood. The initial reports of CrChR1 and CrChR2 described that truncation of the cytoplasmic C-terminal domain did not alter the ion channel function ${ }^{9,10}$. Thus, researchers investigated their molecular properties by using truncated proteins carrying only the 7-TM domain. However, an extensive study of the phosphoproteome of the eyespot fraction of $C$. reinhardtti showed three phosphorylation sites for $\mathrm{CrChR} 1$ and one for $\mathrm{CrChrR2}{ }^{33}$. Thus, a kinase-dependent regulation of function was also proposed. Recently, it has been shown that phosphorylation of CrChR1 is connected to photomotility and calcium signaling of green alga ${ }^{34}$. Predicting the phosphorylation of KnRh3 suggested that several motifs are involved in phosphorylation 
(Fig. S7). It would be interesting to delineate the functional role of these predicted phosphorylation patterns of $\mathrm{KnRh} 3$ in a native system.

We demonstrated that the $\mathrm{C}$-terminus region affects the light-gated ion channel function. The channel closure rate (off-kinetics) slowed down from $10 \mathrm{~ms}$ to $130 \mathrm{~ms}$ as the cytoplasmic domain was shortened (Fig. 2H). In other words, the C-terminus domain accelerates channel closure and the photocycle. Positively charged residues, R287 and R291, in particular, are crucial for the acceleration, because the double mutant R287A/R291A reduced the fast kinetics (Fig. 4F). Furthermore, light sensitivity was altered following systematic truncation of the C-terminus (Fig. 6A and B). Therefore, we propose that an electrostatic interaction exists between the residues of the C-terminus and the 7-TM domain of KnRh3 (Fig. 4I). Although we did not identify the counterparts of R287 and R291 in this study, the candidates could be negatively charged residues such as Asp and Glu in the TM domain, which should be investigated in the future.

Modulation of photocycle kinetics by the cytoplasmic domain has never been proven in ion-transporting rhodopsin thus far. However, in the case of enzyme rhodopsin (HKR or 2C-cyclops), the full-length HKR has been shown to exhibit a normal photocycle which reverts to the initial dark state in $20 \mathrm{sec}$, whereas truncation of the enzyme domain at the $\mathrm{C}$-terminus region resulted in production of a bi-stable photocycle in which rhodopsin possesses two dark states $\left(400 \mathrm{~nm}\right.$ and $380 \mathrm{~nm}$ ), which exhibit photochromism ${ }^{35,36}$. Detailed functional characterization of KnRh3 with spectroscopy and a structural study together with mutation analysis would be needed to reveal the mechanism of the functional modulation by the cytoplasmic domain.

The action spectrum of $\mathrm{KnRh} 3$, assessed from a voltage clamp experiment, revealed its absorption maxima $\left(\lambda_{\max }\right)$ at 430 and $460 \mathrm{~nm}$. To the best of our knowledge, this is one of the most blue-shifted $\lambda_{\max }$ in naturally occurring ChRs. Govorunova et al. previously reported a blue-shifted ChR from Platymonias subcordiformus (PsChR) that displayed an action spectrum of $\lambda_{\max }=445 \mathrm{~nm}$, and an absorption maximum of $437 \mathrm{~nm}$ after purification (in solution) ${ }^{19}$. They ascribed the blue-shift to amino acid residues G142 and A146 in 4-TM (T159 and G163 in CrChR2). In fact, replacement of these residues resulted in a blue shift when tested with $\mathrm{C} 1 \mathrm{C} 2$ and $\mathrm{iC}++{ }^{37,38}$. KnRh3 conserves $\mathrm{A} 146$ (as A161) while G142 is replaced by A157. Thus, A161 would be one of the color determinants in KnRh3, which could be applied to optogenetics. Usually, for optogenetics tools, long-wavelength absorption is ideal for minimizing light scattering by biological tissues. No photocurrent was observed in KnRh3 after illumination with $550 \mathrm{~nm}$ light (Fig. 5). The blue-shifted spectrum of $\mathrm{KnRh} 3$ is ideal for combinatorial applications with long wavelength-absorbing channelrhodopsin or fluorescent indicators to separate the stimulation or excitations.

Given its high homology to $\mathrm{CrChR2}$, KnRh3 exhibits a non-selective cation channel, and permeating monovalent and divalent cations including $\mathrm{H}^{+}, \mathrm{Na}^{+}, \mathrm{K}^{+}, \mathrm{Mg}^{2+}$ and $\mathrm{Ca}^{2+}$. The permeation ratio was similar to that of CrChR2. The cation channelrhodopsin from Cryptophyte algae (DTD channelrhodopsin) exhibited different ratio of cation permeation, in which monovalent metal cations were mainly 
transported with minimum $\mathrm{H}^{+}$and $\mathrm{Ca}^{2+}$ permeation ${ }^{39,40} . \mathrm{KnRh}^{2}$ conducts $\mathrm{Ca}^{2+}$ at a relatively high amount (Fig. 7 and Fig. S6). There is the potential for engineering highly $\mathrm{Ca}^{2+}$ permeable channels, similar to the calcium-permeable ChR2 variant (Catch) ${ }^{22}$. The fast off-kinetics of KnRh3 397 amino acids has great potential for optical neuron excitation with high frequency.

\section{Abbreviations}

ChR, channelrhodopsin

CrChR2, Chlamydomonas reinhardtii Channelrhodopsin-2

KnRh3, Klebsormidium nitens channelrhodopsin

TM, trans-membrane

\section{Declarations}

\section{Acknowledgements}

We thank Miki Iwatani, Ryoko Nakamura and Kyoko Tsunoda for their excellent technical assistance. This work was financially supported by the Japanese Ministry of Education, Culture, Sports, Science and Technology (25104009, 15 H02391 to H.K and 18 K06109 to S.P.T.), a JST CREST grant (JPMJCR1753 to H.K.), and a JST PRESTO grant (JPMJPR1688 to S.P.T).

\section{Conflicts of Interest}

The authors declare no conflicts of interest.

\section{Author contribution}

S.K., S.P.T and H.K. devised the initial idea for the project. K.S and S.S. performed bioinformatic analyses. S.H. performed immunostaining experiments. R.T. performed electrophysiological experiments. S.P.T. prepared the manuscript with contributions from all of the authors to the data analysis, figure generation and the final manuscript.

\section{References}

1. Ernst, O. P. et al. Microbial and animal rhodopsins: Structures, functions, and molecular mechanisms. Chem. Rev. 114, 126-163 (2014).

2. Govorunova, E. G., Sineshchekov, O. A., Li, H. \& Spudich, J. L. Microbial rhodopsins: diversity, mechanisms, and optogenetic applications. Annu. Rev. Biochem. 86, 845-872 (2017). 
3. Kandori, H. Ion-pumping microbial rhodopsins. Front. Mol. Biosci. 2, 52 (2015).

4. Oesterhelt, D. \& Stoeckenius, W. Rhodopsin-like protein from the purple membrane of Halobacterium halobium. Nat. New Biol. 233, 149-152 (1971).

5. Matsuno-Yagi, A. \& Yasuo Mukohata. Two possible roles of bacteriorhodopsin; a comparative study of strains of Halobacterium halobium differing in pigmentation. Biochem. Biophys. Res. Commun. 78, 237-243 (1977).

6. Schobert, B. \& Lanyi, J. K. Halorhodopsin is a light-driven chloride pump. J. Biol. Chem. 257, 1030610313 (1982).

7. Inoue, K. et al. A light-driven sodium ion pump in marine bacteria. Nat. Commun. 4, 1678 (2013).

8. Inoue, K. et al. A natural light-driven inward proton pump. Nat. Commun. 7, 13415 (2016).

9. Nagel, G. et al. Channelrhodopsin-1: a light-gated proton channel in green algae. Science 296, 23952398 (2002).

10. Nagel, G. et al. Channelrhodopsin-2, a directly light-gated cation-selective membrane channel. Proc. Natl. Acad. Sci. U. S. A. 100, 13940-5 (2003).

11. Kato, H. E. et al. Crystal structure of the channelrhodopsin light-gated cation channel. Nature 482, 369-74 (2012).

12. Volkov, O. et al. Structural insights into ion conduction by channelrhodopsin 2. Science 358,6366 (2017).

13. Boyden, E. S., Zhang, F., Bamberg, E., Nagel, G. \& Deisseroth, K. Millisecond-timescale, genetically targeted optical control of neural activity. Nat. Neurosci. 8, 1263-8 (2005).

14. Ishizuka, T., Kakuda, M., Araki, R. \& Yawo, H. Kinetic evaluation of photosensitivity in genetically engineered neurons expressing green algae light-gated channels. Neurosci. Res. 54, 85-94 (2006).

15. Schneider, F., Grimm, C. \& Hegemann, P. Biophysics of channelrhodopsin. Annu. Rev. Biophys. 44, 167-186 (2015).

16. Prigge, M. et al. Color-tuned channelrhodopsins for multiwavelength optogenetics. J. Biol. Chem. 287, 31804-31812 (2012).

17. Wang, H. et al. Molecular determinants differentiating photocurrent properties of two channelrhodopsins from Chlamydomonas. J. Biol. Chem. 284, 5685-5696 (2009).

18. Klapoetke, N. C. et al. Independent optical excitation of distinct neural populations. Nat. Methods 11, 338-46 (2014).

19. Govorunova, E. G., Sineshchekov, O. A., Li, H., Janz, R. \& Spudich, J. L. Characterization of a highly efficient blue-shifted channelrhodopsin from the marine alga Platymonas subcordiformis. J. Biol. Chem. 288, 29911-29922 (2013).

20. Berndt, A., Yizhar, O., Gunaydin, L. A., Hegemann, P. \& Deisseroth, K. Bi-stable neural state switches. Nat. Neurosci. 12, 229-34 (2009).

21. Yizhar, O. et al. Neocortical excitation/inhibition balance in information processing and social dysfunction. Nature 477, 171-178 (2011). 
22. Kleinlogel, S. et al. Ultra light-sensitive and fast neuronal activation with the $\mathrm{Ca}^{2+}$-permeable channelrhodopsin CatCh. Nat. Neurosci. 14, 513-518 (2011).

23. Vierock, J., Grimm, C., Nitzan, N. \& Hegemann, P. Molecular determinants of proton selectivity and gating in the red-light activated channelrhodopsin Chrimson. Sci. Rep. 7, 1-15 (2017).

24. Wietek, J. et al. Conversion of channelrhodopsin into a light-gated chloride channel. Science 344, 409-412 (2014).

25. Berndt, A., Lee, S. Y., Ramakrishnan, C. \& Deisseroth, K. Structure-guided transformation of channelrhodopsin into a light-activated chloride channel. Science 344, 420-4 (2014).

26. Govorunova, E. G., Sineshchekov, O. A., Janz, R., Liu, X. \& Spudich, J. L. Natural light-gated anion channels: A family of microbial rhodopsins for advanced optogenetics. Science 349, 647-650 (2015).

27. Kim, Y. S. et al. Crystal structure of the natural anion-conducting channelrhodopsin GtACR1. Nature 561, 343-348 (2018).

28. Hori, K. et al. Klebsormidium flaccidum genome reveals primary factors for plant terrestrial adaptation. Nat. Commun. (2014). doi:10.1038/ncomms4978

29. Pierangelini, M., Ryšánek, D., Lang, I., Adlassnig, W. \& Holzinger, A. Terrestrial adaptation of green algae Klebsormidium and Zygnema (Charophyta) involves diversity in photosynthetic traits but not in $\mathrm{CO}_{2}$ acquisition. Planta (2017). doi:10.1007/s00425-017-2741-5

30. Buensuceso, R. N. C. et al. Cyclic AMP-independent control of twitching motility in Pseudomonas aeruginosa. J. Bacteriol. (2017). doi:10.1128/JB.00188-17

31. Pushkarev, A. et al. A distinct abundant group of microbial rhodopsins discovered. Nature (2018). doi:10.1038/s41586-018-0225-9

32. Ritter, E., Stehfest, K., Berndt, A., Hegemann, P. \& Bartl, F. J. Monitoring light-induced structural changes of channelrhodopsin-2 by UV-visible and Fourier transform infrared spectroscopy. J. Biol. Chem. 283, 35033-35041 (2008).

33. Wagner, V. et al. The phosphoproteome of a Chlamydomonas reinhardtii eyespot fraction includes key proteins of the light signaling pathway. Plant Physiol. (2008). doi:10.1104/pp.107.109645

34. Böhm, M. et al. Channelrhodopsin-1 phosphorylation changes with phototactic behavior and responds to physiological stimuli in chlamydomonas. Plant Cell (2019). doi:10.1105/tpc.18.00936

35. Tian, Y., Gao, S., Heyde, E. L., Hallmann, A. \& Nagel, G. Two-component cyclase opsins of green algae are ATP-dependent and light-inhibited guanylyl cyclases. BMC Biol. 16, 1-18 (2018).

36. Luck, M. et al. A photochromic histidine kinase rhodopsin (HKR1) that is bimodally switched by ultraviolet and blue light. J. Biol. Chem. 287, 40083-40090 (2012).

37. Kato, H. E. et al. Atomistic design of microbial opsin-based blue-shifted optogenetics tools. Nat. Commun. (2015). doi:10.1038/ncomms8177

38. Wietek, J. et al. Anion-conducting channelrhodopsins with tuned spectra and modified kinetics engineered for optogenetic manipulation of behavior. Sci. Rep. (2017). doi:10.1038/s41598-017- 
14330-y

39. Shigemura, S., Hososhima, S., Kandori, H. \& Tsunoda, S. P. Ion channel properties of a cation channelrhodopsin, Gt_CCR4. Appl. Sci. 9, (2019).

40. Sineshchekov, O. A., Govorunova, E. G., Li, H. \& Spudich, J. L. Bacteriorhodopsin-like channelrhodopsins: Alternative mechanism for control of cation conductance. Proc. Natl. Acad. Sci. 114, E9512-E9519 (2017).

\section{Figures}


A

\begin{tabular}{|l|c|c|c|c|c|c|c|c|c|c|c|c|c|c|}
\hline BR number & 40 & 41 & 48 & 55 & 56 & 59 & 85 & 89 & 90 & 94 & 96 & 118 & 212 & 216 \\
\hline CrChR2 number & 82 & 83 & 90 & 97 & 98 & 101 & 123 & 127 & 128 & 132 & 134 & 159 & 253 & 257 \\
\hline PsChR number & 65 & 66 & 73 & 80 & 81 & 84 & 106 & 110 & 111 & 115 & 117 & 142 & 236 & 240 \\
\hline KnRh3 number & 79 & 80 & 87 & 94 & 95 & 98 & 121 & 125 & 126 & 130 & 132 & 157 & 250 & 254 \\
\hline GtCCR4 number & 67 & 68 & 75 & 89 & 90 & 93 & 116 & 120 & 121 & 125 & 127 & 147 & 242 & 246 \\
\hline TM number in CrChR2 & 2 & 2 & 2 & 2 & 2 & 2 & 3 & 3 & 3 & 3 & 3 & 4 & 7 & 7 \\
\hline BR & K & K & L & T & M & S & D & T & T & L & D & M & D & K \\
\hline CrChR2 & E & E & E & E & F & E & E & T & C & L & H & T & D & K \\
\hline PsChR & E & E & E & E & L & E & E & T & C & L & H & G & D & K \\
\hline KnRh3 & E & V & E & E & I & P & E & T & C & L & A & A & D & K \\
\hline GtCCR4 & Q & E & E & I & T & G & D & T & C & T & D & T & D & K \\
\hline
\end{tabular}

B
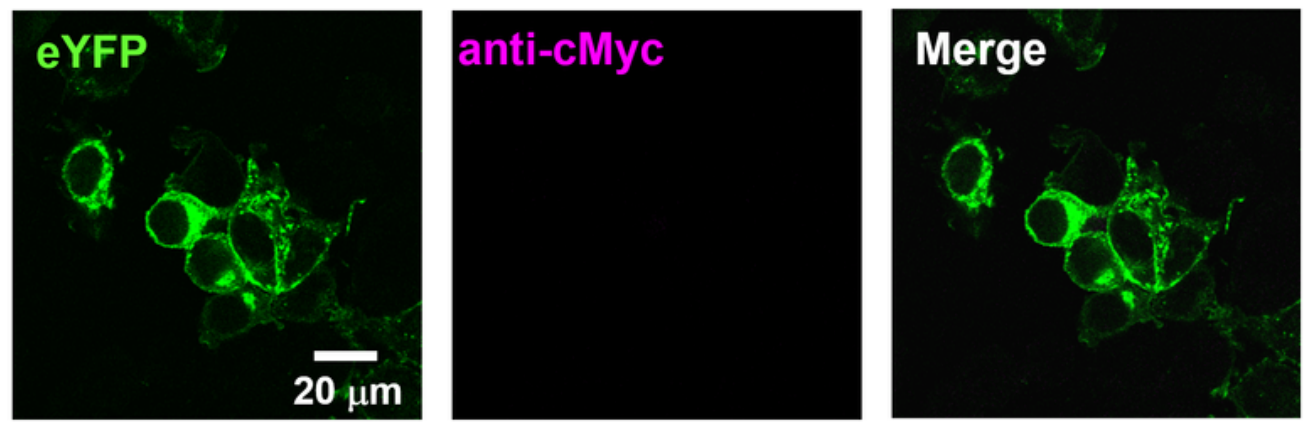

C
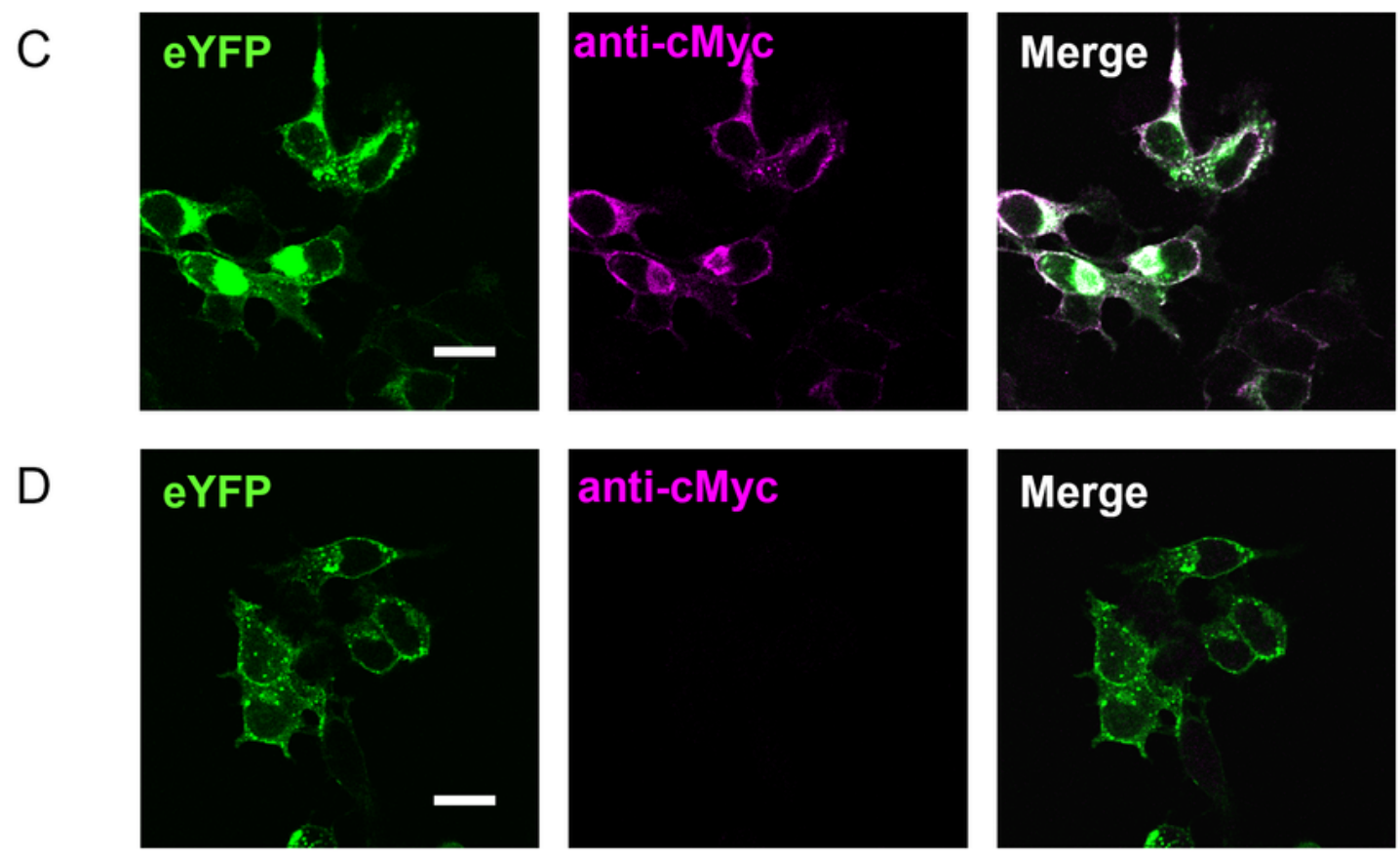

\section{Figure 1}

A, Amino acid alignments of bacteriorhodopsin (BR), CrChR2, KnRh3 and GtCCR4. The characteristic amino acids in BR and CrChR2 were selected. In addition, amino acid numbers of each protein and transmembrane helix (TM) number are indicated. See Fig. S1 for an alignment of the whole protein sequences. B, Immunostaining of KnRh3. Expression of KnRh3-3.0-eYFP-cMyc in cultured ND7-23 cells, with KnRh3 bearing the c-Myc epitope tag at the C-terminus. eYFP fluorescence (left, green), probed with a 
c-Myc antibody under non-permeabilized conditions for immunofluorescent staining with Alexa Fluor 594 (middle, magenta) and merge (right). Scale bar, $20 \mu \mathrm{m}$. C, Expression of KnRh3-3.0-eYFP-cMyc in cultured ND7-23 cells. eYFP fluorescence (left, green), probed with a c-Myc antibody under permeabilized conditions (0.5\% Triton X-100) for immunofluorescent staining with Alexa Fluor 594 (middle, magenta) and merge (right). D, Expression of KfR-3.0-eYFP in cultured ND7-23 cells. eGYP fluorescence (left, green), probed with a c-Myc antibody under permeabilized conditions $(0.5 \%$ Triton X-100) for immunofluorescent staining with Alexa Fluor 594 (middle, magenta) and merge (right).
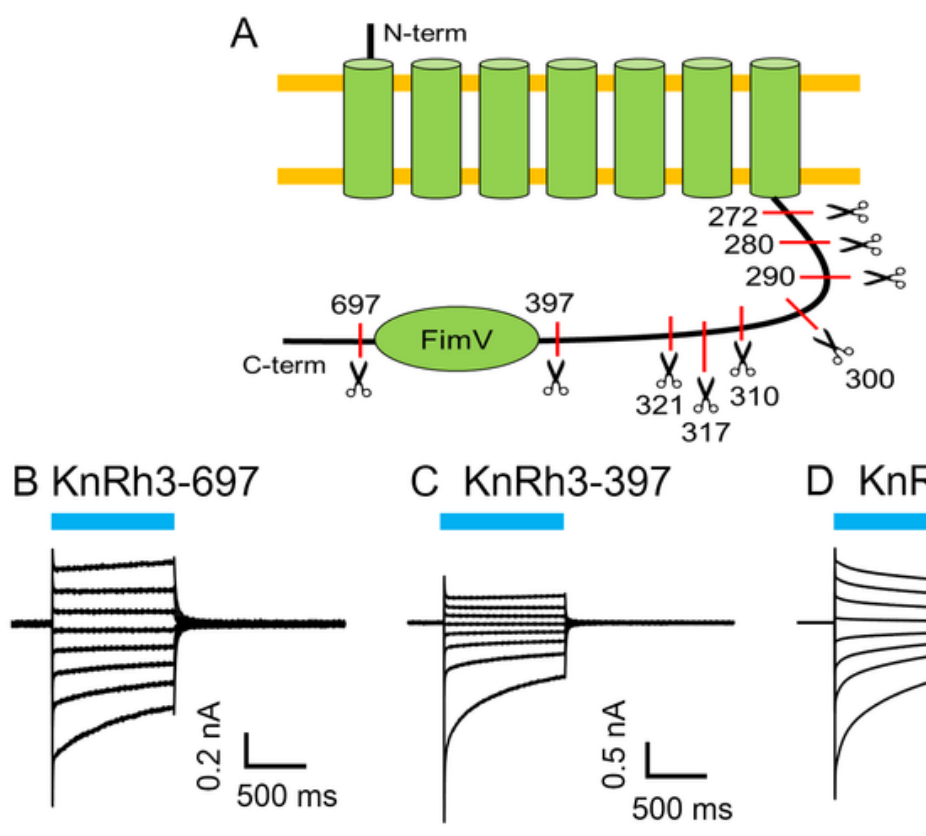

D KnRh3-300
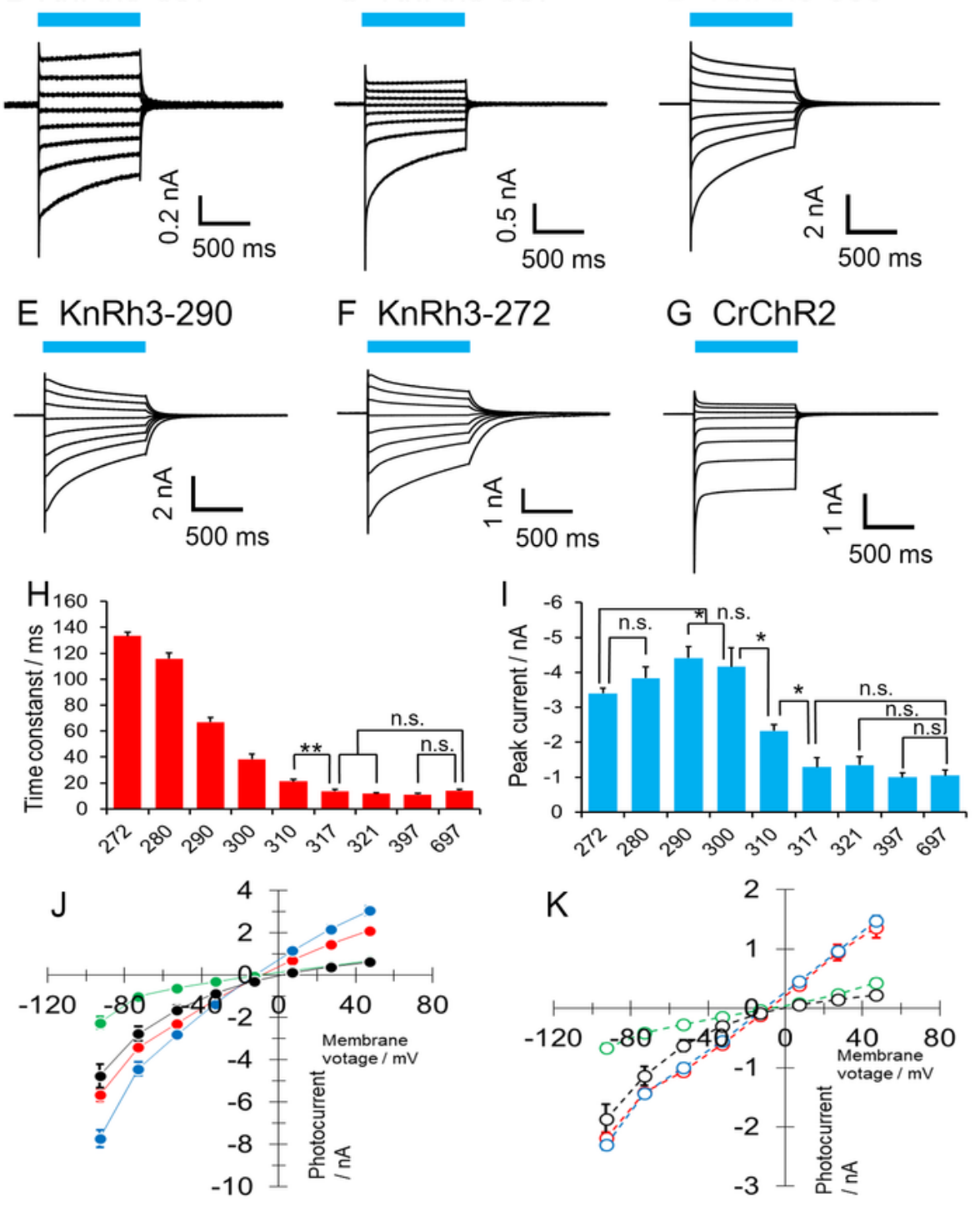
Figure 2

Electrophysiological measurements of various C-terminal length constructs of KnRh3. A, Schematic drawing of truncated constructs. Amino acid positions truncated are indicated. B G, Photocurrent traces of various C-terminal length constructs and CrChR2. Membrane voltage was clamped at from -90 mV to $+50 \mathrm{mV}$ by $+20 \mathrm{mV}$ step. $\mathrm{H}$ and $\mathrm{l}$, Comparison of time constants and photocurrent amplitude from KnRh3 variants. $\mathrm{J}$ and $\mathrm{K}, \mathrm{I}-\mathrm{V}$ relationship of $\mathrm{KnRh} 3$ and $\mathrm{CrChR2}$. Filled and empty symbols indicate peak and steady current, respectively. Red: KnRh3-272, Blue: KnRh3-290, Green: KnRh3-397, Black: CrChR2.
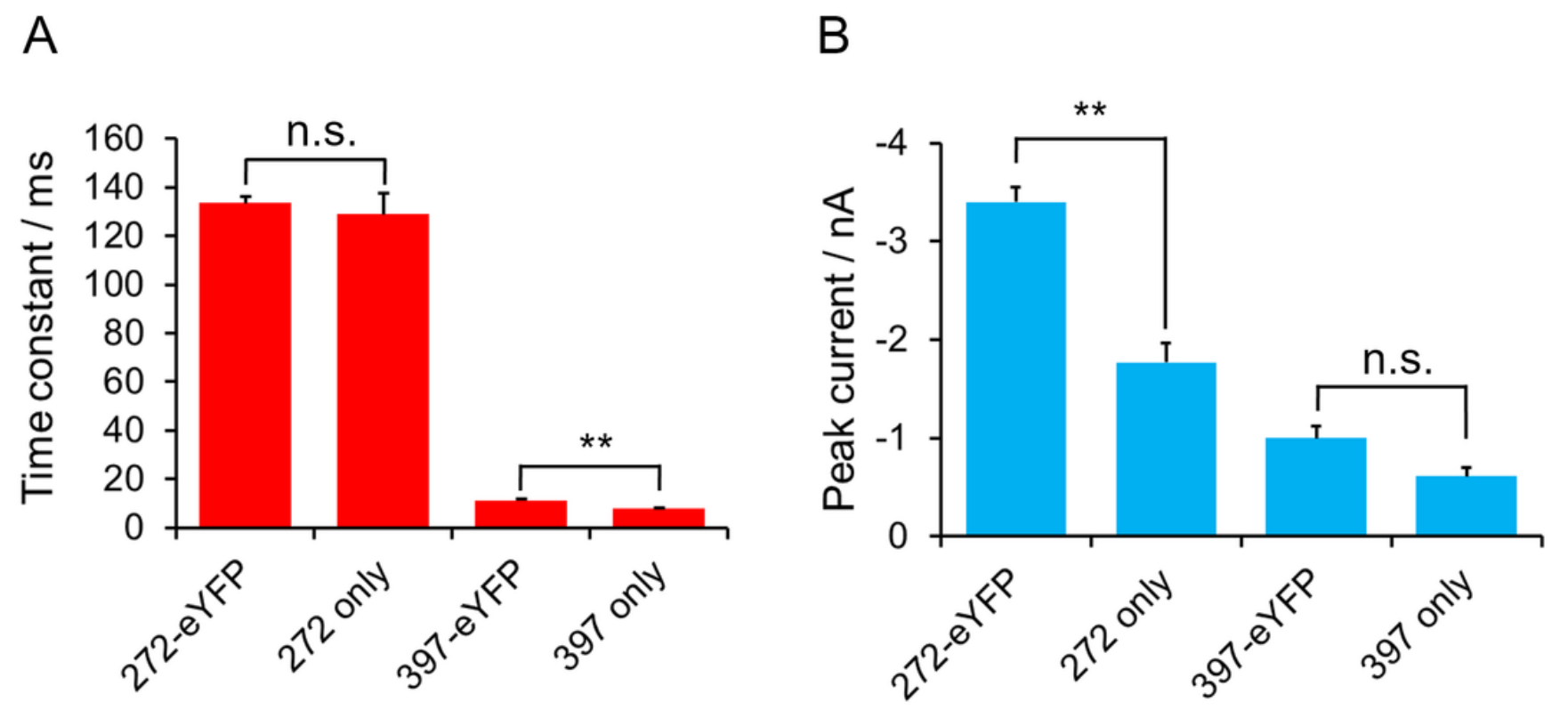

Figure 3

eYFP does not alter the channel kinetics. Time constants (A) and peak current amplitude (B) of KnRh3272 amino acids and -397 amino acids variants with or without fluorescent protein $(N=6-7)$. 

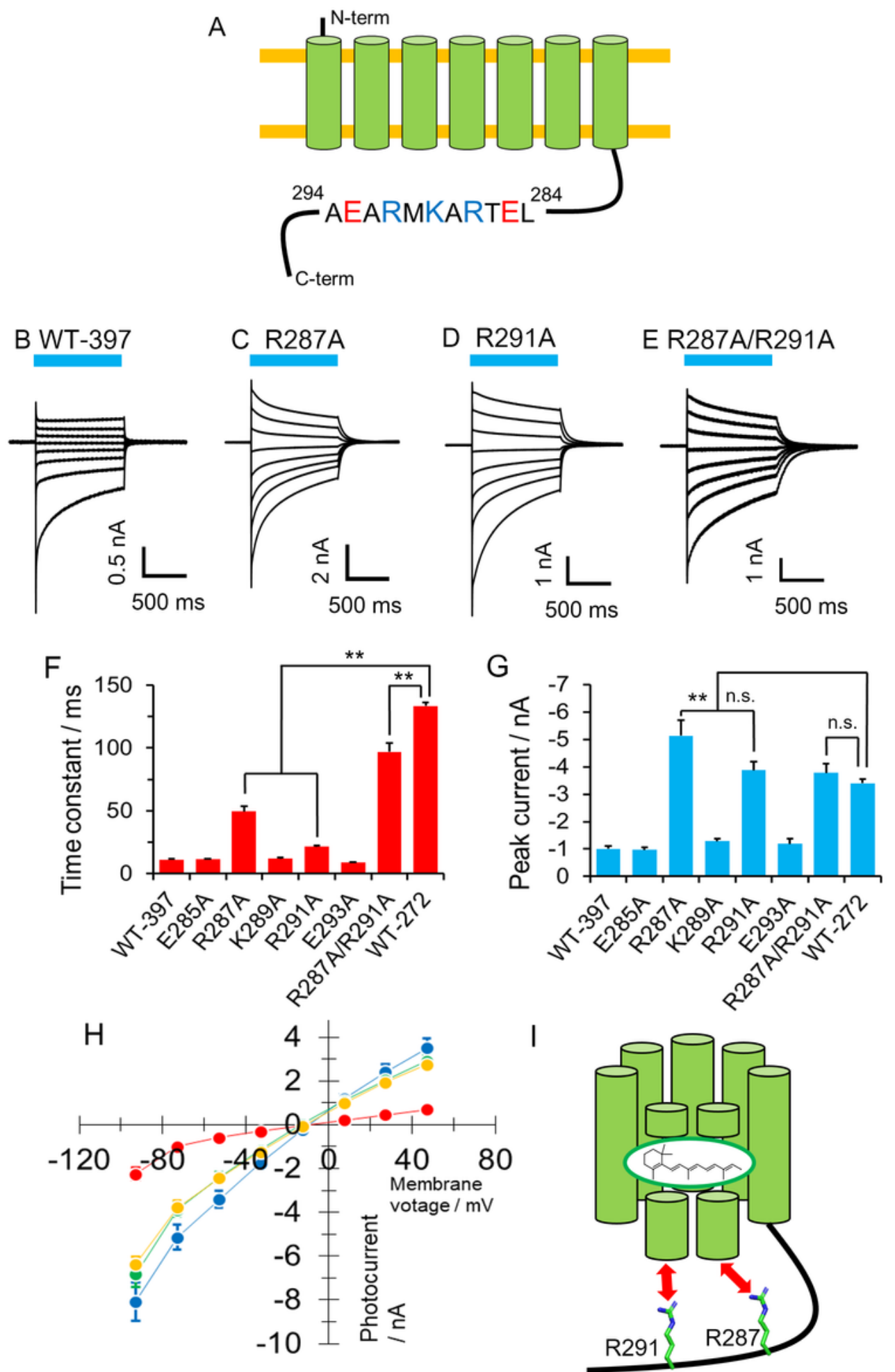

Figure 4

Electrophysiological measurements of variants in the C-terminal region. A, Schematic drawing of KnR3 with amino acid sequence between 284 and 294. B-E, Photocurrent traces of KnRh3-397 (no mutation), mutant variants-R287A, -R291A and -R287A/R291A respectively. Membrane voltage was clamped at from $-90 \mathrm{mV}$ to $+50 \mathrm{mV}$ by $+20 \mathrm{mV}$ step. $\mathrm{F}$ and $\mathrm{G}$, Comparison of time constants and peak current amplitude. 
H, I-V relationship of KnRh3-397 (red), -R287A (blue), -R291A (green), and -R287A/R291A (yellow). I, Schematic drawing of interaction between the 7-TM domain and the cytoplasmic region in $\mathrm{KnRh3}$.

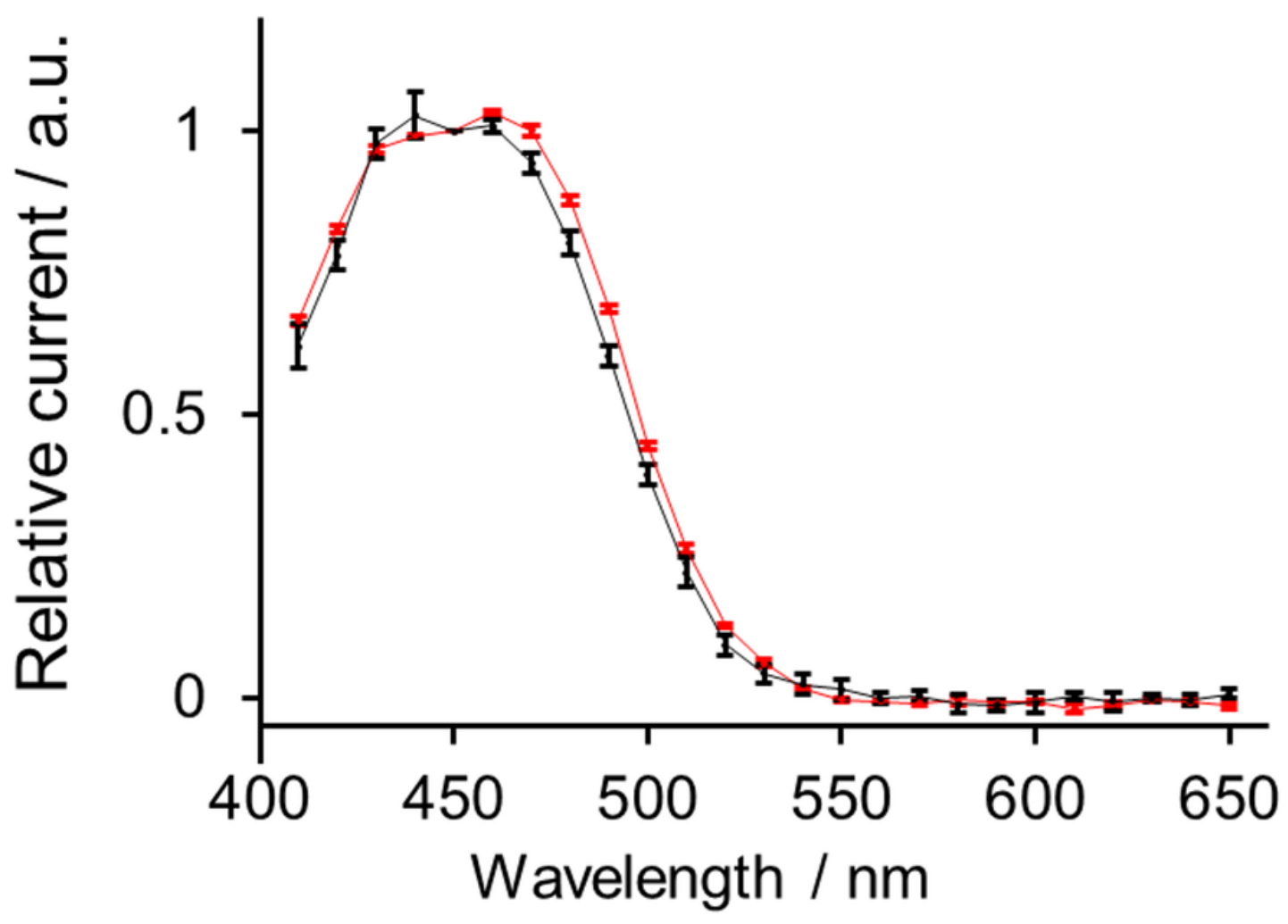

Figure 5

Action spectrum of KnRh3-272 and KnRh-397. Wavelength dependency of the photocurrent. The photocurrents were measured in standard solutions. The membrane voltage was clamped at $-40 \mathrm{mV}$. Red, KnRh3-272, Black, KnRh3-397. N=5. 
A KnRh3-272

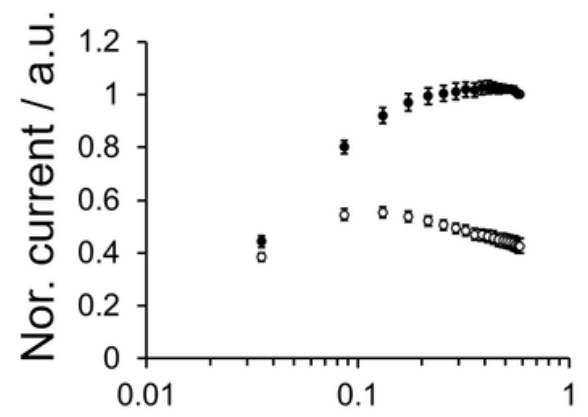

Light intensity / $\mathrm{mWmm}^{-2}$
B KnRh3-397

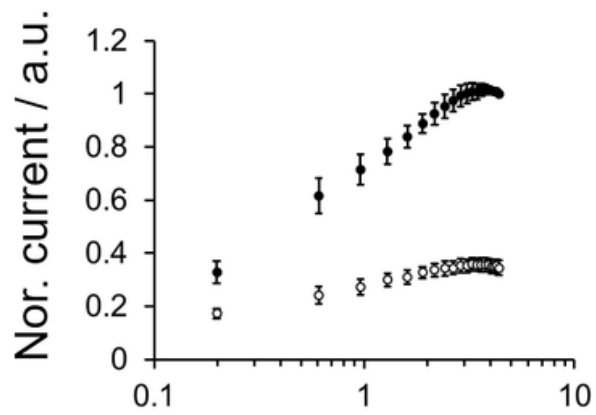

Light intensity / $\mathrm{mWmm}^{-2}$
C CrChR2

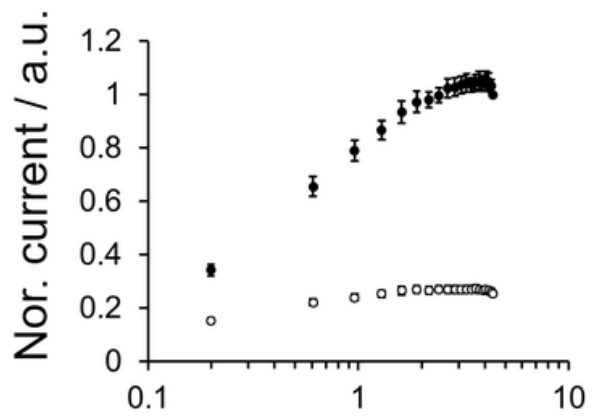

Light intensity / $\mathrm{mWmm}^{-2}$

Figure 6

Light intensity dependence of KnRh3-272 (A), KnRh3-397 (B) and CrChR2 (C). Photocurrent were measured under standard solutions with various light intensities. Membrane voltage was clamped at -60 $\mathrm{mV}$. Black circle, peak current; empty circle, steady state current $(\mathrm{N}=3-4)$. 
A Ex NMG pH9.0

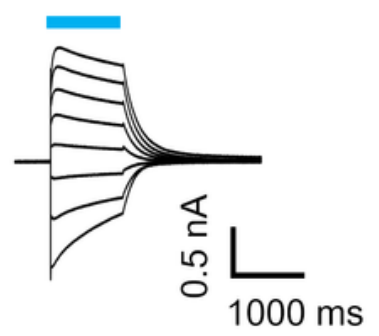

D Ex K+ $\mathrm{KH}^{+} \mathrm{O} .0$
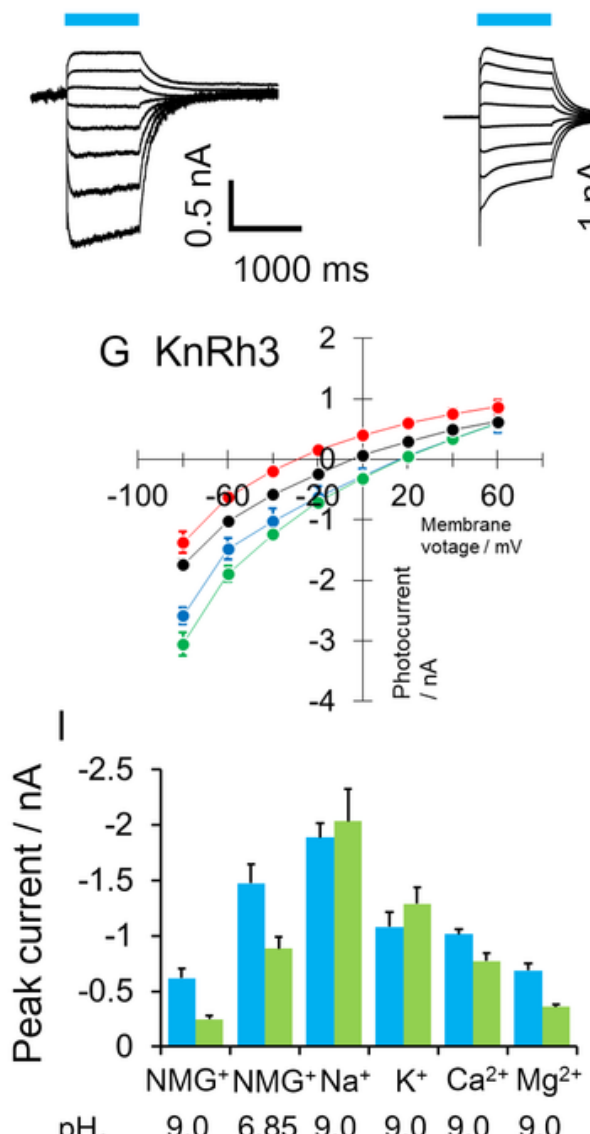

$\begin{array}{lllllll}\mathrm{pH}_{\mathrm{e}} & 9.0 & 6.85 & 9.0 & 9.0 & 9.0 & 9.0\end{array}$

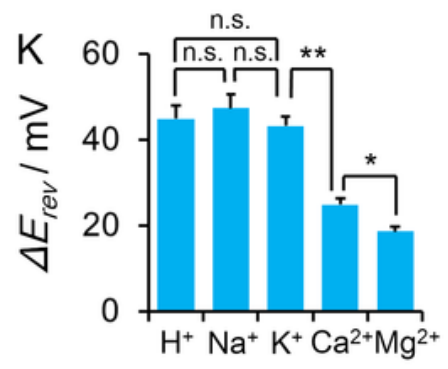

C Ex $\mathrm{Na}^{+} \mathrm{pH} 9.0$

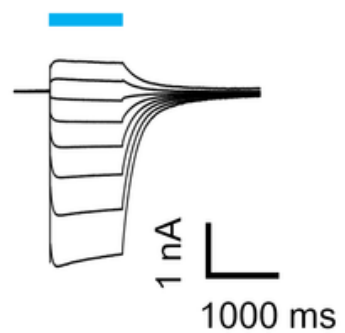

F Ex $\mathrm{Mg}^{2+} \mathrm{pH} 9.0$

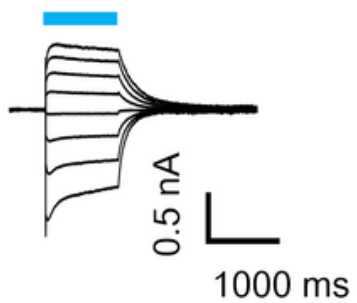

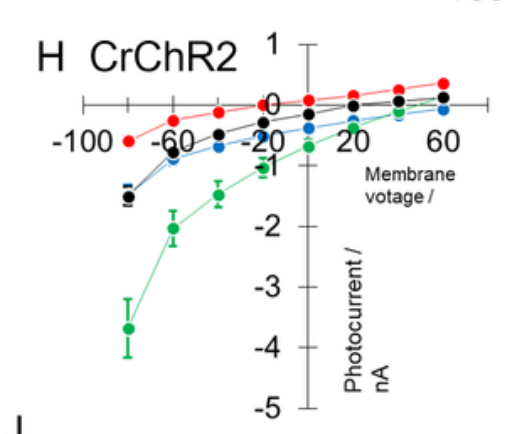
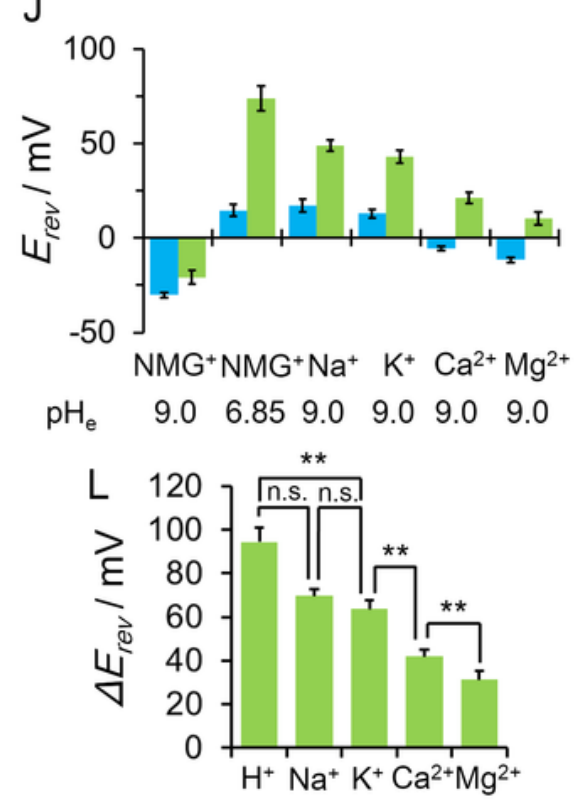

\section{Figure 7}

Ion selectivity of KnRh3 and CrChR2. A-F, Representative photocurrent traces of KnRh3-397 under various cation conditions. Membrane voltage was clamped from at -80 to $+60 \mathrm{mV}$ by $20 \mathrm{mV}$ steps. I-V relationship of KnRh3 $(\mathrm{G})$ and $\mathrm{CrChR2}(\mathrm{H})$. Peak component of photocurrent were plotted. Red: Ex NMG pH 9.0, Blue: Ex NMG pH 6.85, Green: Ex Na+ pH 9.0, Black: Ex Ca2+ pH 9.0. I, Comparison of peak current amplitude under various ionic conditions at -60 mV. light blue, KnRh3; green CrChR2. J, Reversal potential 
Erev under various solutions. $\mathrm{K}$, Reversal potential shift ( $\Delta \mathrm{Erev})$ of $\mathrm{KnRh3}$. $\Delta$ Erev of each cation was calculated by subtracting Erev of each ionic condition in panel $\mathrm{J}$ by Erev of NMG+ at pHe 9.0. e.g. $\Delta$ Erev of $\mathrm{H}+$ is determined as Erev (NMG+ pHe 6.85) - Erev (NMG+ pHe 9.0). L, Reversal potential shift ( $\Delta$ Erev) of CrChR2.

\section{Supplementary Files}

This is a list of supplementary files associated with this preprint. Click to download.

- Fig.STashiro200820.pdf 